\title{
FRACTURE ANALYSIS OF THERMAL BARRIER COATING SYSTEMS UNDER THERMAL SHOCK
}

\author{
ZHANG YAN-YAN*,***, SHEN RI-LIN**, HUANG KAI**, "YUE XIAO-NING* \\ *Department of Physics, Shenyang University, 110000 Shenyang, China \\ **School of Astronautic, Harbin Institute of Technology, 150001 Harbin, China \\ ***Shenyang National Laboratory for Material Sciences, Institute of Metal Research, \\ Chinese Academy of Sciences, 110000 Shenyang, China \\ "E-mail: yuexn0@163.com
}

Submitted July 15, 2016; accepted October 24, 2016

\begin{abstract}
Keywords: Thermal barrier coatings (TBCs), Interaction energy integral method (IEIM), Transient thermal stress intensity factors (TSIFs), Crack growth law

In order to understand the thermal fracture properties of thermal barrier coating (TBC) systems, transient thermal stress intensity factors (TSIFs) are calculated by using the transient interaction energy integral method (IEIM) when a thermal shock load is applied. In this study, continuous and weak interfaces are studied, considering different distributions of the material properties and their derivatives in the interface. The effect of the interface bonding strength on TSIFs at crack tip of TBC systems with multiple interfaces is analysed. This method provides an avenue to simulate interfacial properties subjected to thermal shock loads.
\end{abstract}

\section{INTRODUCTION}

Thermal barrier coating (TBC) systems [1], with good thermal insulation performance, typically have an interface or multiple interfaces, characterized by nonhomogeneous material properties across the interface. Particularly, TBCs with bonding layers can be considered as multiple interface nonhomogeneous materials. Thermal expansion coefficients mismatch between the coating and the substrate may cause interface debonding of the coating-substrate system [2]. The thermal fracture resistance of TBCs has attracted enormous attention due to its wide applications in engineering [3]. Fracture parameters of nonhomogeneous materials with interfaces have been widely studied in order to understand the thermal fracture behavior of TBC systems. Lee and Erdogan [4] investigated interface cracking of functionally graded material (FGM) coatings under loading by the finite element method. Li and Lee [5] developed a model to study a mode III crack problem when a coating crack is inclined to the functionally graded coating/substrate interface which is weak or micro-discontinuous. By the domain-independent interactive energy integral method (IEIM), Guo et al. [6] investigated FGMs with a single crack subjected to steady-state thermal loading. Zhang et al. [7] studied the interface properties across nonhomogeneous coating/substrate materials and the effect of the interface discontinuity and geometric parameter on the transient thermal stress intensity factors
(TSIFs). Petrova and Schmauder [8] explored a bimaterial, composed of a homogeneous material and a FGM with small internal cracks, to understand the underlying thermal fracture mechanism. Petrova and Schmauder [9] further studied the fracture processes in the vicinity of an interface crack in functionally graded/homogeneous bimaterials with internal defects under tensile loading and a heat flux. Ding and Li [10] analyzed the fracture behavior of functionally graded layered structures under thermal loading, with an interface crack. Yang et al. [11] investigated the interfacial cracking process in TBCs subjected to compression by acoustic emission and digital image correlation methods. Song et al. [12] studied the crack problem at interface between TBCs and metal substrates. Wang et al. [13] calculated the transient temperature fields and the dynamic stress intensity in TBCs by finite element methods. Li et al. [14] investigated the behavior of the temperature fields near the crack and the stress intensity factors when an inner crack is parallel to the heated surface of a thermoelastic strip under thermal shock. Shang et al. [15] showed how an arbitrarily oriented crack affects the heat transfer properties of an infinite functionally graded medium under uniform remote heat flux. Guo et al. [16] studied the behavior of lanthanum zirconate-based TBCs under thermal shock and thermal cycling loads. However, research on the thermal shock fracture problem in nonhomogeneous materials with multiple interfaces is still quite limited up to now. 


\section{THEORETICAL}

Problem formulation

In this paper we analyze the thermal shock crack problem in TBC systems. In Figure 1 a schematic of a TBC system with two interfaces is shown. The thickness of the whole system is $h$ and the normalized crack length of TBC systems is $a / h$, while the thicknesses of the coating, bonding layer and substrate are $h_{1}, h_{2}$ and $h_{3}$, respectively. The Young's modulus, density, specific heat, coefficient of thermal expansion, and heat conductivity are defined as $E(x), \rho(x), c(x), \alpha(x)$ and $\lambda(x)$. The thermomechanical properties of the TBC system are distributed along the $x$-direction. Subscripts 1 and 3 are used for denoting the coating (ceramic) material and the substrate (metal) material, respectively. In Figure 1, two types of interfaces are included: 1. a strong interface, material properties and their derivatives are continuous on the interface (interface between ceramic and bonding layer); 2. a weak interface, whose material properties are continuous, but its derivatives are discontinuous on the interface (interface between metal and bonding layer). The initial temperature condition is $T_{0}$ and sudden temperature changes are considered for both surfaces of the plate with $T_{1}=T_{3}=T$. The normalized time is defined as $t_{n}=\lambda_{l} t /\left(c_{l} \rho_{l} h^{2}\right)$. The transient thermal stress intensity factors(TSIFs)willbenormalized by $K_{0}=\sqrt{ } \square \square \pi \square a E_{1} \alpha_{1} T_{1} /$ $/(1-v)$

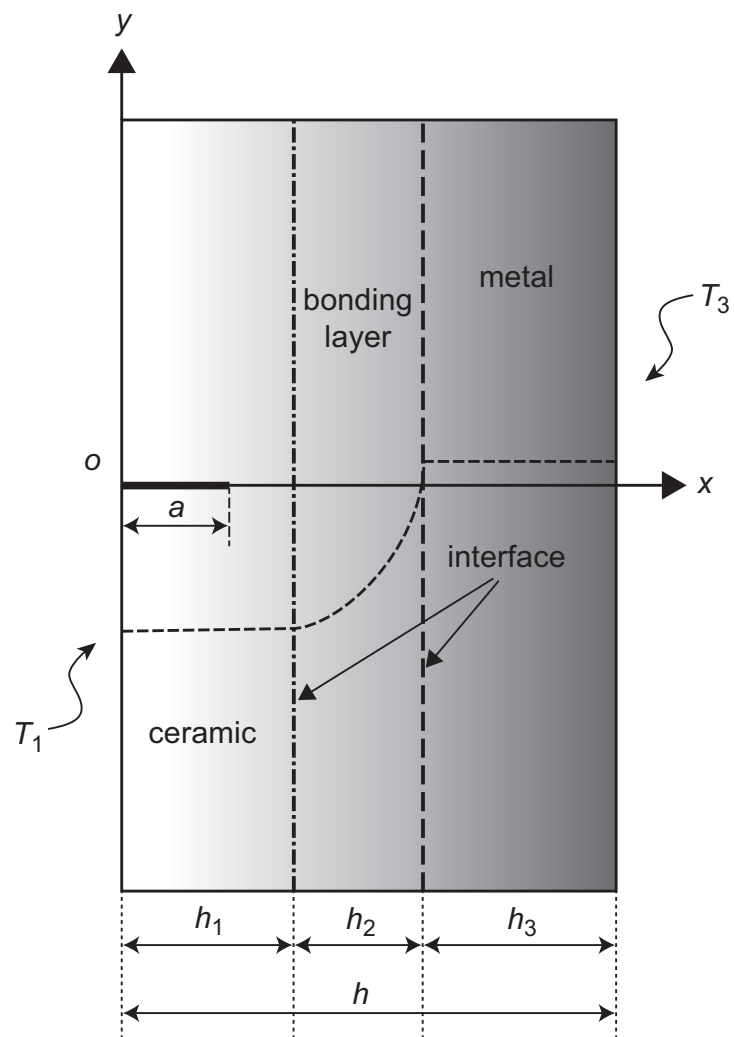

Figure 1. Schematic of a TBC system with two interfaces.
Determination of the temperature fields through FDM-FEM

Temperature fields can be determined by using finite differential and finite element methods (FDM-FEM). Referring to the TBC system in Figure 1, the transient temperature field is denoted as $T(x, t)$. It is assumed that the process of heat transfer is adiabatic. In order to obtain the temperature field in the TBC system without any crack we can write the unsteady one-dimensional heat conduction equation as:

$$
\frac{\partial}{\partial x}\left[\lambda(x) \frac{\partial T(x, t)}{\partial x}\right]=C(x) \rho(x) \frac{\partial T(x, t)}{\partial t} .
$$

Thus, the finite element equation of the transient temperature field [7] can be expressed as:

$$
\begin{aligned}
& \left(\left[K_{1}\right]+\left[K_{2}\right]+\frac{2}{\Delta t}\left[K_{3}\right]\right)\{T(x, t)\}_{n+1}= \\
& =\left(-\left[K_{1}\right]-\left[K_{2}\right]+\frac{2}{\Delta t}\left[K_{3}\right]\right)\{T(x, t)\}_{n}+\{P\}_{n}+\{P\}_{n+1} .
\end{aligned}
$$

where $\left[\mathrm{K}_{1}\right]$ is the thermal matrix, $\left[\mathrm{K}_{2}\right]$ the convection matrix, $\left[\mathrm{K}_{3}\right]$ the heat capacity matrix, and $\{\mathrm{P}\}$ the thermal load vector.

The transient IEIM and extraction of the transient TSIFs

The stress intensity factor characterizes the crack tip stress field strength, but for nonhomogeneous materials it is very difficult to obtain. The IEIM is a very convenient method to obtain the TSIFs with high efficiency. Kc and Kim [18] illustrated the finite element evaluation of nonsingular T-stress and mixed-mode TSIFs within nonhomogeneous materials under steady-state thermal load condition by the interaction integral method. Yu et al. developed an IEIM for mechanical and thermal fracture problems of nonhomogeneous materials with different mechanical properties [19-23]. In this paper, when the

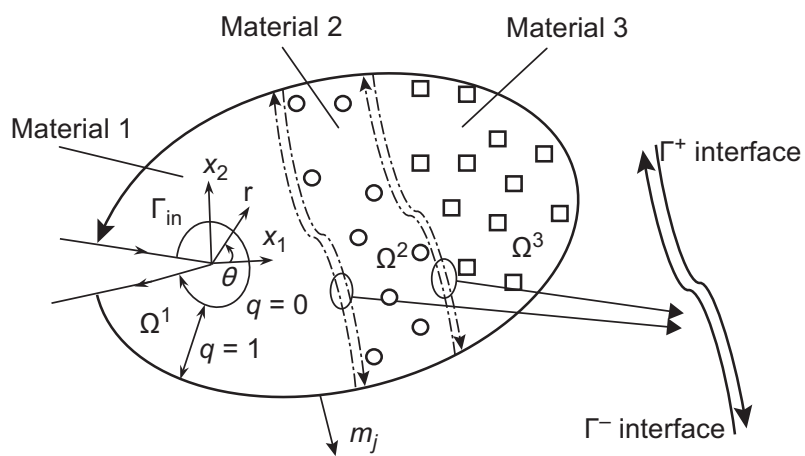

Figure 2. The integral domain with two interfaces for the IEIM at the crack tip. 
integral domain contains multiple interfaces, according to the literature $[7,23]$ the IEIM can be written as:

$$
I(t)=I_{\text {mechanical }}(t)+I_{\text {temperature }}(t)+I_{\text {interface }}(t)
$$

where the first and second integrals are the mechanical and thermal parts of the interaction energy release rate, and the third integral is a line integral along the interfaces which vanishes for continuous material properties.

A schematic of the integral domain with multiple interfaces is shown in Figure 2. The detailed domain expression of the modified transient IEIM with multiple interfaces for the TBCs is $[7,25]$ :

$$
\begin{aligned}
I(t)= & \int_{\Omega}\left[\sigma_{i j}(t) u_{i, 1}^{a u x}+\sigma_{i j}^{a u x} u_{i, 1}(t)-\sigma_{i k}^{a u x} \varepsilon_{i k}(t) \delta_{1 j}\right] q_{, j} d \Omega \\
& +\int_{\Omega} \sigma_{i j} t \quad S_{i j k l}^{t i p}-S_{i j k l} x \quad \sigma_{i k, 1}^{a u x} q d \Omega \\
& +\sum_{1}^{n} \int_{\Omega^{n}} \sigma_{i i}^{a u x}\left\{\alpha_{, 1}^{n}\left[T(t)-T_{0}\right]+\alpha T(t)_{, 1}^{n}\right\} q d \Omega \\
& \left.\left.+\sum_{1}^{n-1} \int_{\Gamma_{\text {interface }}^{n-1}} \sigma_{i k}^{a u x} t \quad \alpha^{n}-\alpha^{n-1} \quad T-T_{0}\right) \cos \theta_{n-1}\right] q d \Gamma
\end{aligned}
$$

In Equation 4 set $n=3$, which means that there are three separate integral domains divided by two interfaces in the TBC system. The relation between $I(\mathrm{t})$ [24] and the mixed-mode transient TSIFs is

$$
I(t)=2\left[K_{I}(t) K_{I}^{a u x}(t)+K_{I I}^{a u x}(t)\right] / E_{t i p}^{\prime},
$$

where $K_{I}(t)$ and $K_{I I}(t)$ are mode-I TSIFs and mode-II TSIFs, respectively. The TSIFs marked by the superscript aux denote the auxiliary TSIFs. For plane strain $E_{t i p}^{\prime}=$ $=E_{t i p}^{\prime} /\left(1-v_{t i p}^{2}\right)$, for plane stress $E_{t i p}^{\prime}=E_{t i p}^{\prime}$.

Let $K_{I}^{a u x}(t)=1$ and $K_{I I}^{a u x}(t)=0$, and the mode-I TSIF can be extracted as

$$
K_{I}(t)=E_{t i p}^{\prime} I(t) / 2 .
$$

Similarly, the mode-II TSIF can be obtained as

$$
K_{I I}(t)=E_{t i p}^{\prime} I(t) / 2 .
$$

Thus, the mixed-model transient TSIFs can be determined.

\section{Verification}

In order to verify the present method when considering the interface discontinuity, we compare the verification example results with those in Ref. [6]. All the distributions of the properties are defined by exponential functions. The normalized TSIFs are calculated for an embedded crack shown in Figure 3. The following data are used for numerical analysis, with the thermal expansion coefficient $\alpha$ defined as:

$$
\begin{aligned}
& \text { Case A: } \begin{cases}\alpha(x)=\alpha_{1} \times e^{2 \delta x} & (x \leq 0.5 W) \\
\alpha(x)=\alpha_{1} \times e^{\delta} & (x>0.5 W)\end{cases} \\
& \text { Case B: } \begin{cases}\alpha(x)=\alpha_{1} \times e^{2 \delta x} & (x \leq 0.5 W) \\
\alpha(x)=5 \alpha_{1} & (x>0.5 W)\end{cases}
\end{aligned}
$$

Where $L / W=2,2 a / W=0.2, \theta=0, v=0.33, \delta=\ln (2) / W$

$$
\begin{gathered}
E(x)=E_{1} \times e^{\delta x} \\
\lambda(x)=\lambda_{1} \times e^{\delta x}
\end{gathered}
$$

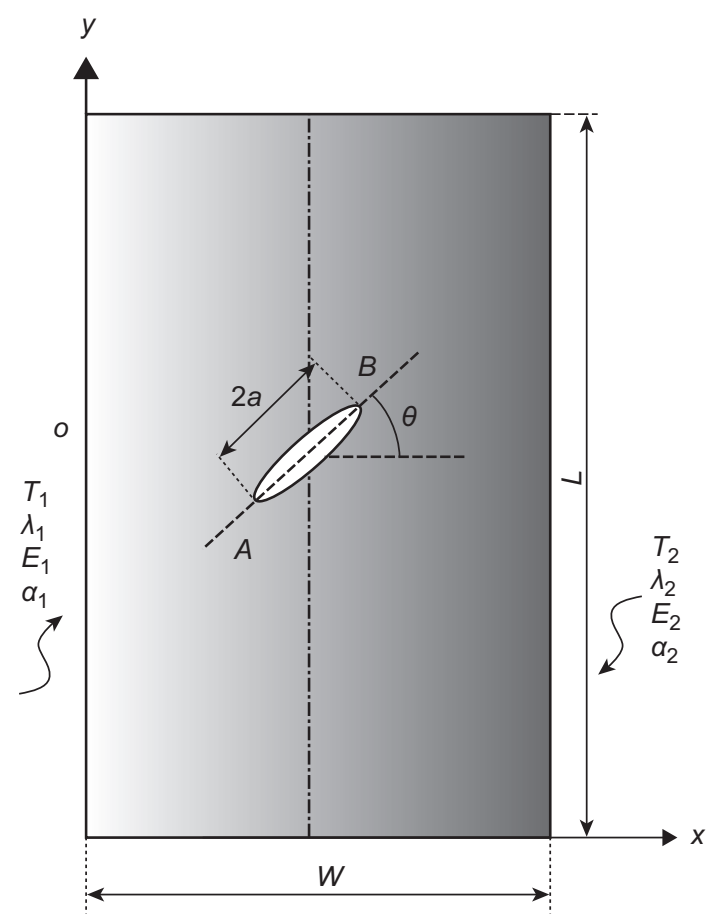

Figure 3. An inclined crack in a nonhomogeneous plate with an interface.

Table 1. Comparison between the present TSIFs and the corresponding analytical results in Ref. [6].

\begin{tabular}{ccc}
\hline \multicolumn{3}{c}{ Results in Ref. [6] } \\
\hline Length crack $a$ & \multicolumn{2}{c}{ TSIFs } \\
\cline { 2 - 3 }$a$ & Case B \\
\hline 0.28921 & 0.07861 & 0.51887 \\
0.40014 & -0.0597 & 1.00943 \\
0.54882 & -0.32041 & 0.09559 \\
0.58986 & -0.34906 & -1.06604 \\
0.59319 & -0.34906 & -1.40566 \\
0.59785 & -0.34906 & -1.69497 \\
0.6037 & -0.34906 & -2.18553 \\
0.6483 & -0.27358 & -1.88364 \\
0.68828 & -0.21069 & -1.58176 \\
\hline \hline
\end{tabular}

\begin{tabular}{ccc}
\hline \hline & Present results \\
\hline Length crack & Case A & Case B \\
\cline { 2 - 3 }$a$ & \multicolumn{2}{c}{ TSIFs } \\
\hline 0.29 & 0.07586 & 0.51381 \\
0.402 & -0.07629 & 1.02622 \\
0.549 & -0.30804 & 0.12208 \\
0.59 & -0.3315 & -1.05506 \\
0.595 & -0.33015 & -1.37213 \\
0.598 & -0.3284 & -1.6652 \\
0.602 & -0.32422 & -2.17938 \\
0.649 & -0.26448 & -1.86644 \\
0.69 & -0.20937 & -1.57613 \\
\hline
\end{tabular}


The result is shown in Table 1, where the parameters used in the example are identical to those given in Reference [6]. It can be found from Table 1 that the relative deviations between the present results and those in Reference [6] are all within $1.5 \%$. It can thus be concluded that the present method is efficient and accurate to solve thermal crack problems in TBC systems with discontinuous material properties and material property gradients.

\section{RESULTS AND DISCUSSIONS}

We note that the thermomechanical properties of the bonding layers can be illustrated by general functions in the current method. The $x$-dependent thermomechanical properties of the bonding layers of the TBC are shown in Figure 1.

The material parameters are listed in Table 2 and the thermomechanical properties of the TBC systems are assumed as:

$E(x)=E_{1}, \alpha(x)=\alpha_{1}, \lambda(x)=\lambda_{1}, c(x)=c_{1}, \rho(x)=\rho_{1}$

$\left(0<x<h_{1}\right)$

$$
\begin{gathered}
E(x)=E_{1} e^{\left(\beta_{1} / h_{1}\right) x}, \alpha(x)=\alpha_{1} e^{\left(\beta_{2} / h_{1}\right) x}, \lambda(x)=\lambda_{1} e^{\left(\beta_{3} / h_{1}\right) x}, \\
c(x)=c_{1} e^{\left(\beta_{4} / h_{1}\right) x}, \rho(x)=\rho_{1} e^{\left(\beta_{5} / h_{1}\right) x} \\
\left(h_{1}<x<h_{2}\right) \\
E(x)=E_{3}, \alpha(x)=\alpha_{3}, \lambda(x)=\lambda_{3}, c(x)=c_{3}, \rho(x)=\rho_{3} \\
\left(h_{3}<x<h\right)
\end{gathered}
$$

The geometry parameters are taken to be $h_{1}=0.2 h$, $h_{2}=0.1 h$ and $h_{3}=0.7 h$ in the simulations, and we conduct all the simulations under plane strain state. The nonhomogeneity constants are defined as

$$
\begin{gathered}
\beta_{1}=\ln \left(E_{2} / E_{1}\right), \beta_{2}=\ln \left(\alpha_{2} / \alpha_{1}\right), \beta_{3}=\ln \left(\lambda_{2} / \lambda_{1}\right), \\
\beta_{4}=\ln \left(c_{2} / c_{1}\right), \beta_{5}=\ln \left(\rho_{2} / \rho_{1}\right),
\end{gathered}
$$

Figures 4 and 5 show the temperature and thermal stress field distributions, respectively, at different normalized times in the TBC system. It is found that the effect of interface properties on the temperature and thermal stress fields becomes more significant with time. The change of the temperature field is also directly related to the material inhomogeneity.

Figure 6 shows the variation of the TSIFs with respect to the normalized time for different normalized crack lengths $(0.1,0.2,0.3$ and 0.4 , respectively).

Table 2. Thermomechanical properties of TBC systems [17].

\begin{tabular}{lcccccc}
\hline Material & $\begin{array}{c}\text { Specific heat } \\
\left(\mathrm{J} \cdot \mathrm{kg}^{-1} \cdot \mathrm{K}^{-1}\right)\end{array}$ & $\begin{array}{c}\text { Thermal conductivity } \\
\left(\mathrm{W}(\mathrm{mK})^{-1}\right)\end{array}$ & $\begin{array}{c}\text { Coefficient of thermal } \\
\text { expansion }\left(\times 10^{-6} / \mathrm{K}\right)\end{array}$ & $\begin{array}{c}\text { Density } \\
\left(\mathrm{kg} \cdot \mathrm{m}^{-3}\right)\end{array}$ & $\begin{array}{c}\text { Young's modulus } \\
(\mathrm{GPa})\end{array}$ & $\begin{array}{c}\text { Poisson's } \\
\text { ratio }\end{array}$ \\
\hline Ceramic & $c_{1}=456.7$ & $\lambda_{1}=2.09$ & $\alpha_{1}=10.0$ & $\rho_{1}=5331$ & $E_{1}=151.0$ & $v_{1}=0.33$ \\
Metal & $c_{3}=537.0$ & $\lambda_{3}=7.5$ & $\alpha_{3}=9.5$ & $\rho_{3}=4420$ & $E_{3}=116.7$ & $v_{3}=0.33$ \\
\hline
\end{tabular}
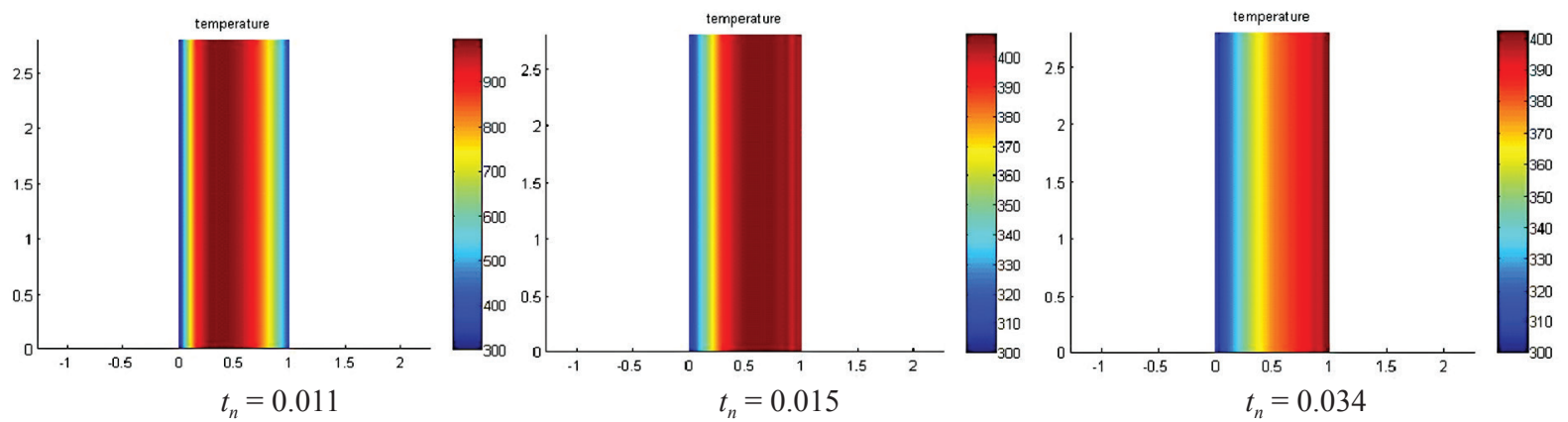

Figure 4. Evolution of the temperature field in the TBC system.
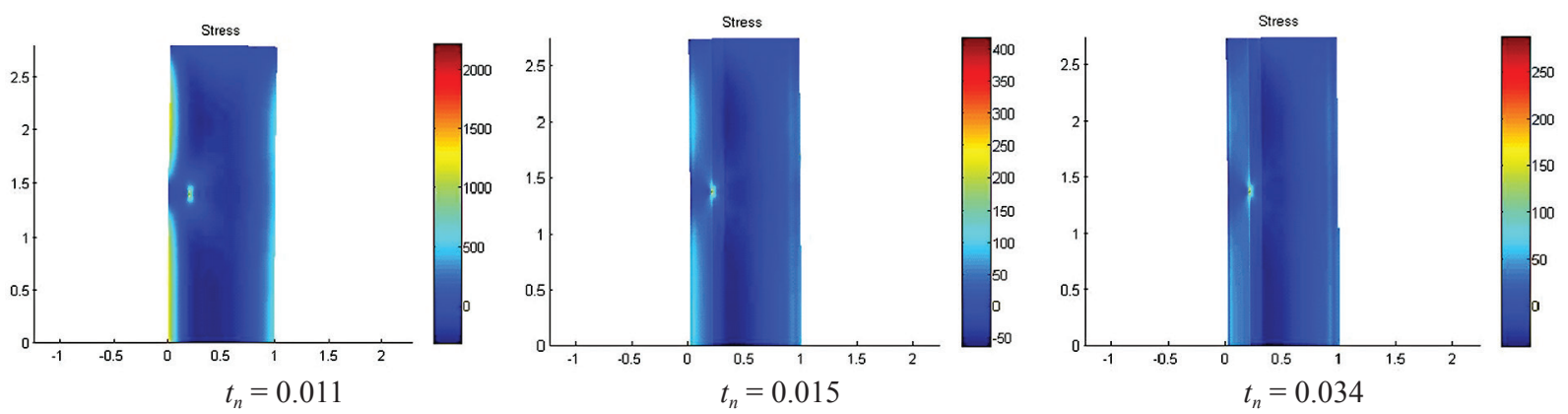

Figure 5. Evolution of the thermal stress field in the TBC system. 


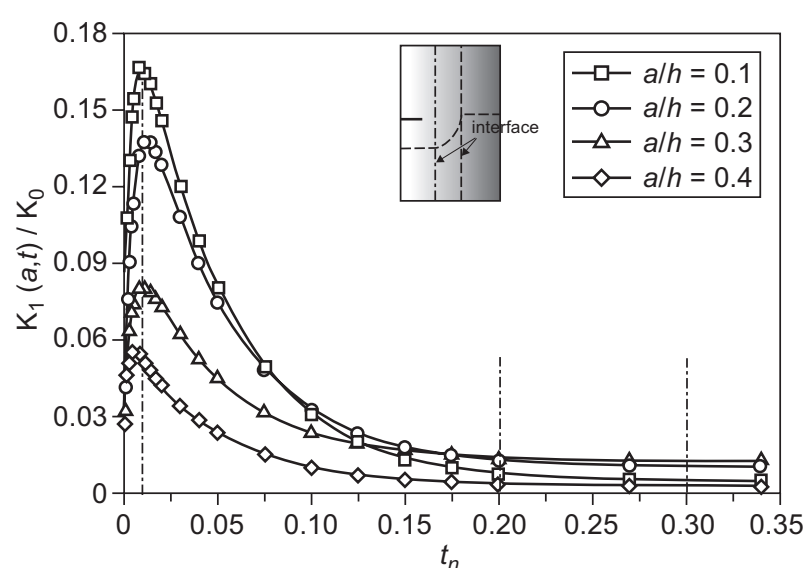

Figure 6. Transient TSIFs versus normalized time for different crack lengths.

The transient TSIF at the early stage of thermal shock is a dominant factor to fracture failure as shown in Figure 6. It can be observed that the peak values of the TSIFs are affected by the normalized crack length. The peak values of the TSIFs change in a regular way, but the variation trend of the TSIFs with respect to the crack length is irregular for longer times. When the interfaces are touched by the crack tip, the interface properties have different effects on the TSIFs (see Figure 7). Figure 7 shows normalized transient TSIFs versus normalized crack length at different time instants $(0.011,0.2$ and 0.3 ). As mentioned before we have considered two types of interfaces in the TBC system of this paper. It is found that the effect of interface properties on TSIFs under thermal shock are extremely obvious, and that the interface properties of the TBC system will directly affect the TSIFs. Therefore, it is absolutely essential to take the interface discontinuity into account in order to increase the thermal shock resistance of the system when designing TBC systems. Using the method proposed in this paper, we are able to describe the situation that the material properties and its derivatives are discontinuous.

\section{CONCLUSIONS}

In this paper, the thermal shock fracture problem of TBC systems with two interfaces is studied. The results show that: 1. The transient IEIM is an efficient method to obtain the TSIF in a TBC system with two interfaces; 2. The transient TSIF at the early stage of thermal shock is a dominant factor to fracture failure; 3 . The TSIFs are affected differently by the interface discontinuity and the normalized crack lengths.

\section{Acknowledgements}

The work is supported by NSFC (11072067), the General Project for Department of Liaoning Education (No. L2015364).

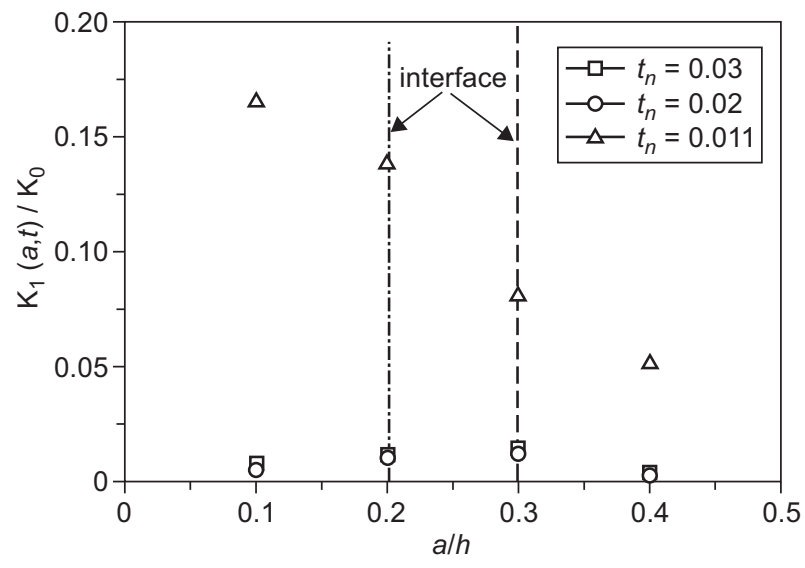

Figure 7. Normalized transient TSIFs versus normalized crack length at different time instants.

\section{REFERENCES}

1. Zhou H., Li F., He B. (2006): Research progresses in materials for thermal barrier coatings. Materials Review, 20(6), 40-43.

2. Tian Y.S., Chen C.Z., Liu J.H. (2005): Research development of $\mathrm{ZrO}_{2}$ thermal barrier coatings. China Mechanical Engineering, 16(16), 1499-1503.

3. Zeng A.X., Tang S Q. (1999): Preparation application and development of metallic-matrix ceramic coating. Surface Technology, 28(1), 1-3.

4. Lee Y.D., Erdogan F. (1998): Interface cracking of FGM coatings under steady-state heat flow. Engineering Fracture Mechanics, 59(3), 361-380. doi:10.1016/S0013-7944 (97)00137-9

5. Li Y. D., Lee K.Y. (2009): Effects of the weak/microdiscontinuity of interface on thefracture behavior of a functionally graded coating with an inclined crack. Archive of Applied Mechanics, 79(9), 779-791. doi:10.1007/s00419008-0252-3

6. Guo L. C., Guo F. N., Yu H. J., Zhang L. (2012): An interaction energy integral method for nonhomogeneous materials with interfaces under thermal loading. International Journal of Solids and Structures, 49(2), 355-365. doi:10.1016/j.ijsolstr.2011.10.012

7. Zhang Y.Y., Guo L.C., Guo F.N., Zhong. S.Y. (2012): Fracture analysis of a nonhomogeneous coating/substrate system with an interface under thermal shock. Acta Mechanica, 225(9), 2485-2500. doi:10.1007/s00707-0141087-3

8. Petrova V., Schmauder S. (2011): Crack-interface crack interactions in functionally graded/homogeneous composite bimaterials subjected to a heat flux. Mechanics of Composite Materials, 47(1),125-136. doi:10.1007/s11029-011-9191-0

9. Petrova V., Schmauder S. (2012): Interaction of a system of cracks with an interface crack in functionally graded/ homogeneous bimaterials under thermo-mechanical loading. Computational Materials Science, 64, 229-233. doi:10.1016/j.commatsci.2012.04.032

10. Ding S. H., Li X. (2011): Thermal stress intensity factors for an interface crack in a functionally graded layered structures. Archive of Applied Mechanics, 81(7): 943-955. doi:10.1007/s00419-010-0433-8 
11. Yang L., Zhong Z.C., Zhou Y.C. (2016): Acoustic emission assessment of interface crackingin thermal barrier coatings. Acta Mechanica Sinica, 32(2), 342-348. doi:10.1007/ s10409-015-0514-6

12. Song G.R., Yan T.T., He C. F., Yang S., Lu Y., Wu B. (2015): Detection of surface crack on the substrate under thermal barriercoatingsusing microwavenon-destructive evaluation. Journal of Microwave Power and Electromagnetic Energy, 49(2), 69-75. doi:10.1080/08327823.2015. 11689897

13. Wang L., Li D.C.,Yang J.S. (2016): Modeling of thermal properties and failure of thermal barrier coatings with the use of finite element methods: A review. Journal of the European Ceramic Society, 36, 1313-1331. doi:10.1016/j. jeurceramsoc.2015.12.038

14. Li W., Song F., Abdelmoula, R., Li J. (2016): Non-Fourier effect and inertia effect analysis of a strip with an induced crack under thermal shock loading. Engineering Fracture Mechanics, 162, 309-323. doi:10.1016/j. engfracmech. 2016.02.056

15. Shang W. T., Tz C. C., Ching H. C. (2014): Temperature distribution and heat flow around a crack of arbitrary orientation in a functionally graded medium. Journal of Engineering Mathematics, 87,123-137. doi:10.1007/ s10665-013-9664-3

16. Guo X. Y., Zhe L., Jung Y. G. Thermal properties, thermal shock, and thermal cycling behavior of lanthanum zirconate-based thermal barrier coatings. Metallurgical and Materials Transactions E. doi:10.1007/s40553-016-0070-4

17. Noda N., Guo L.C. (2008): Thermal shock analysis for a functionally graded plate with a surface crack. Acta Mechanica, 195, 157-166. doi:10.1007/s00707-007-0562-5

8. Kc A., Kim J.H. (2008): Interaction integrals for thermal fracture of functionally graded materials. Engineering
Fracture Mechanics, 75(8), 2542-2565. doi:10.1016/j.engfracmech. 2007.07.011

19. Yu H.J., Wu L.Z., Guo L.C., He Q. L., Du S.Y. (2010): Interaction integral method for the interfacial fracture problems of two nonhomogeneous materials. Mechanics of Materials, 42(4), 435-450. doi:10.1016/j. mechmat.2010.01.001

20. Yu H.J., Wu L.Z., Guo L.C., Du S.Y., He Q.L. (2009): Investigation of mixed-mode stress intensity factors for nonhomogeneous materials using an interaction integral method. International Journal of Solids and Structures, 46, 3710-3724. doi:10.1016/j.ijsolstr.2009.06.019

21. Yu H.J., Wu L.Z., Guo L.C., Wu H.P., Du S.Y. (2010): An interaction integral method for $3 \mathrm{D}$ curved cracks in nonhomogeneous materials with complex interfaces. International Journal of Solids and Structures, 47, 21782189. doi:10.1016/j.ijsolstr.2010.04.027

22. Yu H.J., Sumigawa T., Wu L.Z., Kitamura T. (2015): Generalized domain-independent interaction integral for solving the stress intensity factors of nonhomogeneous materials. International Journal of Solids and Structures, 67-68, 151-168. doi:1016/j.ijsolstr.2015.03.035

23. Yu H.J., Kitamura T. (2015): A new domain-independent interaction integral for solving the stress intensity factors of the materials with complex thermo-mechanical interfaces. European Journal of Mechanics A/Solids, 49, 500-509. doi:10.1016/j.euromechsol.2014.09.007

24. Zhang Y.Y., Guo L.C., Noda N. (2013): Investigation methods for thermal shock crack problems of functionally graded materials-Part II: combined analytical-numerical method. Journal of Thermal Stresses, 37, 325-339. doi:10.1 080/01495739.2013.839854 